Journal of Animal and Veterinary Advances 11 (14): 2529-2532, 2012

ISSN: $1680-5593$

(C) Medwell Journals, 2012

\title{
Effects of Grape Seed Extract on Renal Ischemic Reperfusion in Rats
}

\author{
${ }^{1,2}$ Mehrdad Hashemi, ${ }^{3}$ Yousef Doustar, ${ }^{1}$ Fataneh Hashem Dabaghian and \\ ${ }^{1}$ Seyed Ashrafadin Goushegir \\ ${ }^{1}$ Research Institute for Islamic and Complementary Medicine, \\ Tehran University of Medical Sciences, Tehran, Iran \\ ${ }^{2}$ Department of Genetics, Islamic Azad University, Tehran Medical Branch, Tehran, Iran \\ ${ }^{3}$ Department of Pathology, Faculty of Veterinary Medicine, Islamic Azad University, \\ Tabriz Branch, Tabriz, Iran
}

\begin{abstract}
Grape Seed Extract (GSE) has been reported to exert protective effects on various forms of renal ischemic reperfusion disorders. Natural Grape Seed Extract (GSE) are potent free radical scavengers and hence, provide significant protection against oxidative stress. Accordingly, the present study focused on investigating the possible protective role of GSE against ischemic reperfusion mediated damage in renal tissues in rats. The results revealed that oral administration of $250 \mathrm{mg} \mathrm{kg}^{-1}$ (body weight) of GSE for 3 days significantly protect against renal cell apoptosis via the induction of endogenous antioxidant $(\mathrm{p}<0.001)$.
\end{abstract}

Key words: Ischemic reperfusion, grape seeds extract, apoptosis, lipid, protection, Iran

\section{INTRODUCTION}

Acute Renal Failure (ARF), classically defined as an abrupt decrease in kidney function that leads to accumulation of nitrogenous wastes such as blood urea nitrogen and creatinine is a common clinical problem with increasing incidence, serious consequences, unsatisfactory therapeutic options and an enormous financial burden to society (Bagchi et al., 1990, 2000; Das and Maulik, 1994; Shi et al., 2003; Sato et al., 1999). ARF may be classified as prerenal (functional response of structurally normal kidneys to hypoperfusion), intrinsic renal (involving structural damage to the renal parenchyma) and postrenal (urinary tract obstruction). This review focuses on intrinsic ARF which has emerged as the most common and serious subtype in hospitalized patients and can be associated pathologically with Acute Tubular Necrosis (ATN). Consequently, it still is common clinical practice to use the terms intrinsic ARF and ATN interchangeably. Despite decades of pioneering basic research and important technical advances in clinical care, the prognosis for patients with intrinsic $\mathrm{ARF}$ remains poor with a mortality rate of $40-80 \%$ in the intensive care setting. Two major problems have plagued the field and hindered progress. First, well $>20$ definitions for ARF have been used in published studies, ranging from dialysis requirement to subtle increases in serum creatinine (Ray et al., 2000). In an attempt to standardize the definition and reflect the entire spectrum of the condition, the term Acute Kidney Injury (AKI) has been proposed (Bagchi et al., 2000). AKI refers to a complex disorder that comprises multiple causative factors and occurs in a variety of settings with varied clinical manifestations that range from a minimal but sustained elevation in serum creatinine to anuric renal failure. Prerenal azotemia and other fully reversible causes of acute renal insufficiency are specifically excluded from the spectrum of AKI. An inherent shortcoming of this term is the continued reliance on serum creatinine measurements and the definition of AKI undoubtedly will undergo enhancements as novel early biomarkers for the identification of ARF before the rise in serum creatinine come to light (Puiggros et al., 2005). This review avoids the term ATN and uses the expressions AKI and intrinsic ARF transposably. The second problem is an incomplete understanding of the cellular and molecular mechanisms that underlie AKI. This review updates the reader on current advances in basic and translational research that hold promise in human ischemic AKI. Classic concepts are mentioned briefly as founding principles but expanded on only if contemporary findings substantiate or refute them. The reader is referred to recent publications that address the mechanisms that underlie other causes of intrinsic AKI such as sepsis (Fan and Lou, 2004) and nephrotoxins (Bagchi et al., 2003). However, from the clinical viewpoint, it is acknowledged that AKI is

Corresponding Author: Mehrdad Hashemi, Department of Genetics, Islamic Azad University, Tehran Medical Branch, Tehran, Iran 
frequently multifactorial with concomitant ischemic, nephrotoxic and septic components and with overlapping pathogenetic mechanisms. Reperfusion of the ischemic renal tissue is associated with adramatic inflammatory response leading to TNF-alpha $(\mathrm{TNF}-\alpha)$ release, Interleukin-10 (IL-10) induction and subsequent neutrophil-mediated cytotoxic injury (Bagchi et al., 1990). Grape seeds are waste products of the winery and grape juice industry. These seeds contain lipid, protein, carbohydrates and 5-8\% polyphenols depending on the variety. Polyphenols in grape seeds are mainly flavonoids including gallic acid, the monomeric flavan-3-ols catechin, epicatechin, gallocatechin, epigallocatechin and epicatechin 3-O-gallate and dimeric, trimeric and even more polymeric proanthocyanidins (Formigli et al., 1998). A large number of studies have been conducted on GSE and have demonstrated excellent free-radical scavenging and cardioprotective properties (Buttke and Sandstrom, 1994). GSE provided unique protection against renal ischemia-reperfusion injury, renal infarction in rats (Das and Maulik, 1994) and doxorubicin-induced nephrotoxicity in mice (Nakagawa et al., 2005). In a recent study, it was concluded that GSE prevents oxidative injury of HepG 2 cells (a hepatocarcinoma cell line) by modulating the expression of antioxidant enzyme systems (Wei et al., 2011). This study was undertaken to investigate the protective effects of grape seed on apoptotic cell death of renal tissue during experimental renal Ischemia-Reperfusion (I/R) in rats.

\section{MATERIALS AND METHODS}

Animals: Adult male Wistar rats $(200-220 \mathrm{~g})$ were procured from the animal house facility at Tehran Medical Branch, Islamic Azad University. All animals were housed

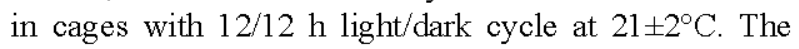
animals were given Purina rat chow and water ad libitum. The animals were kept under observation for 1 week prior to the start of treatment. All animal experiments were carried out in accordance with Tehran Medical Branch, Islamic Azad University Ethical Committee Acts.

Chemicals: Powdered leaves of grape seed were extracted three times with $1 \mathrm{~L}$ of $70 \%$ methanol $(\mathrm{MeOH}) / \mathrm{H}_{2} 0$ while being macerated at room temperature for $24 \mathrm{~h}$ each time. The hydroalcoholic extracts were combined and concentrated in vacuo to yield dried extract and it was defined by the producer as containing $0.28 \%$ hypericin. This hydroalcoholic extract was kept in refrigerator for all experiments.

Induction of ischemic reperfusion: Animals were anesthetized with Ketamine hydrochloride (Ketamine 10\%, Alfasan, Woerden-Holland, $50 \mathrm{mg} \mathrm{kg}^{-1}$ ) and Xylazine
(Xylazin 2\%, Alfasan, Worden-Holland, $5 \mathrm{mg} \mathrm{kg}{ }^{-1}$ ) intraperitoneally. A midline incision was made in each rat and the left kidney became available. Ischemia-reperfusion injury was induced by applying a noncrushing microvascular clamp on the left renal artery for $60 \mathrm{~min}$. After $60 \mathrm{~min}$ of ischemia, the clamp was removed and the tissue was closed in layers. The animals were then returned to their cages, reperfusion period was 3 days after surgery. In experimental group, $3 \mathrm{~h}$ before ischemia, grape seed extract and $60 \mathrm{mg} \mathrm{kg}^{-1}$ were administrated as orally form. Sham-operated animals underwent the same operation but without clamping. Animals were sacrificed after 3 days postoperatively under general anesthesia with an injection of over dosage of Thiopental sodium (60 $\mathrm{mg} \mathrm{kg}^{-1}$ ) and the left kidney was harvested.

Experimental design: Studies were performed on male Wistar rats weighing 200-250 g $(n=40)$. Rats received a standard diet and water ad libitum and were housed in a $12 \mathrm{~h}$ light/dark cycle. The animals were randomly allocated into three groups:

- $\quad$ I/R-saline group in which rats were subjected to renal ischemia for $60 \mathrm{~mm}(\mathrm{n}=10)$

- IR-Grape seed (GSE) group in which rats were administered GSE (250 $\mathrm{mg} \mathrm{kg}^{-1}$ and gavages) $3 \mathrm{~h}$ prior to I/R and 3 day IR $(\mathrm{n}=10)$

- Sham-operated group in which rats were subjected to identical surgical procedure without occlusion of both renal pedicles and maintained under anesthesia for the duration of the experiment $(n=10)$

- Intact group

Determination of renal apoptosis: Apoptosis was evaluated via the Terminal Deoxynucleotidyl Transferase Mediated dUTP Nick-End Labeling (TUNEL) Method with the use of in situ Cell Death Detection kit, POD (1684817, Roche, Germany) according to manufacturer's instructions with some modifications (Bagchi et al., 2000). Briefly, the tissue sections were dewaxed and rehydrated by heating at $60^{\circ} \mathrm{C}$ followed by washing in xylene and rehydration through a graded series of ethanol and double distillated water. Then, the sections were incubated for $30 \mathrm{~min}$ at $21-37^{\circ} \mathrm{C}$ with Proteinase $\mathrm{K}$ working solution $\left(20 \mu \mathrm{g} \mathrm{mL}^{-1}\right.$ in $10 \mathrm{mM}$ Tris-Cl, $\mathrm{pH} \mathrm{7.6).} \mathrm{The}$ sections were rinsed with PBS and incubated with the TUNEL reaction mixture for $1 \mathrm{~h}$ at $37^{\circ} \mathrm{C}$ in a humidified chamber. As a positive control, sections were treated with DNase I ( $1 \mathrm{mg} \mathrm{mL}^{-1}$; Sigma) for $10 \mathrm{~min}$ to introduce nicks in the genomic DNA. After converter Peroxidase (POD) was added, the sections were incubated for $30 \mathrm{~min}$ at $37^{\circ} \mathrm{C}$ in a humidified chamber. Then, the 3,3-diaminobenzidine substrate was added for the visualization of nuclei with DNA nick end labeling. The sections were counter- 
stained with toluidine blue to show normal nuclei. The percentage of renal cells with DNA nick end labeling was analyzed by counting the cells exhibiting brown nuclei at $\mathrm{x} 40$ magnification in 5 randomly chosen fields $\left(1 \mathrm{~mm}^{2}\right)$ in triplicate plates.

The number of TUNEL-positive renal cells was counted by double-blinded observation. The quantitative data for the present study were shown in the form of (mean \pm SEM) and the significant differences between the groups were analyzed using ANOVA and Tukey. The differences were significant at $\mathrm{p}<0.05$.

Statistical analysis: The results were analyzed by oneway ANOVA test followed by Tukey's multiplecomparison post hoc test. The results were expressed as mean \pm SEM, $n=10$. The $\mathrm{p}<0.05$ were considered to be statistically significant.

\section{RESULTS}

Apoptosis: Results of the effect of graded doses of GSE on the incidence rate of apoptotic cell death of renal tubular cells induced by ischemia-reperfusion. In IR group, ischemia/reperfusion+GSE group caused mild apoptotic changes of renal tubular cells as apoptotic cells were observed in light to dark brown color. The incidence rate of apoptotic cell death in this group was significantly higher than Sham/IR group ( $p<0.001)$. In Group 3, GSE $\left(250 \mathrm{mg} \mathrm{kg}^{-1}\right)$ significantly $(\mathrm{p}<0.05)$ reduced apoptosis of renal tubular cells induced by ischemia-reperfusion compared to IR group. However, even this dosage of the GSE could not normalize the changes in apoptosis and there still was a significant difference between this group and IR/Sham $(\mathrm{p}<0.05)$. The numbers of apoptotic cells were significantly higher $(23 \%)$ in the renal cells in $\mathbb{R}$ rat compared to the control group. GSPE treatment significantly reduced the number of apoptotic cells compared to the ischemic/reperfused renal tissue.

\section{DISCUSSION}

The data presented revealed a marked protective effect of GSE against renal ischemic/reperfusion-induced elevation of cell death level in renal tissue. The effect of GSE on NO was recently studied by JohnsonVarghese (Nakagawa et al., 2005). Their study demonstrated that GSE offered protection against hyperoxic effects on renal tissue of rat. Furthermore, pre-treatment with grape seed extract cytoprotective effect in rat type renal proximal tubular cells and podocyte (Wei et al., 2011). A series of studies were conducted using GSE to demonstrate its nephroprotective ability in animals and humans. GSE supplementation improved renal functional assessment including post-ischemic left ventricular function, reduced renal infarct size (Yanarates et al., 2008). The present study demonstrates that GSE, a potent cytoprotective effects can exert anti-apoptotic effects by preserving renal tissue. The data presented provide additional benefits of GSE administration and may offer a promising natural and safe new trend for the prevention of renal ischemic/reperfusion complications.

\section{CONCLUSION}

The study suggests that GSE are effective in ameliorating the damage to renal tissue in experimental ischemic reperfusion. Such effect may be related to their potent antioxidant properties as evidenced by the increase in renal tissue GSH and reduction of lipid peroxidation as well as total nitrate/nitrite levels.

\section{ACKNOWLEDGEMENT}

This research was partially supported by a Research Grant provided by Tehran Medical Branch, Islamic Azad University, Tehran, Iran.

\section{REFERENCES}

Bagchi, D., C.K. Sen, S.D. Ray, D.K. Das, M. Bagchi, H.G. Preuss and J.A. Vinson, 2003. Molecular mechanisms of cardioprotection by a novel grape seed proanthocyanidin extract. Mutation Res. Fundam. Mol. Mech. Mutagen., 523-524: 87-97.

Bagchi, D., D.K. Das, R.M. Engelman, M.R. Prasad and R. Subramanian, 1990. Polymorphonuclear leucocytes as potential source of free radicals in the ischaemicreperfused myocardium. Eur. Heart J., 11: 800-813.

Bagchi, D., M. Bagchi, S.J. Stohs, D.K. Das and S.D. Ray et al., 2000. Free radicals and grape seed proanthocyanidin extract importance in human health and disease prevention. Toxicology, 148: 187-197.

Buttke, T.M. and P.A. Sandstrom, 1994. Oxidative stress as a mediator of apoptosis. Immunol. Today, 15: 7-10.

Das, D.K. and N. Maulik, 1994. Evaluation of antioxidant effectiveness in ischemia/reperfusion tissue injury methods. Methods Enzymol., 233: $601-610$.

Fan, P.H. and H.X. Lou, 2004. Effects of polyphenols from grape seeds on oxidative damage to cellular DNA. Mol. Cell Biochem., 267: 67-74.

Formigli, L., L. Ibba-Manneschi, A.M. Perna, C. Nediani, P. Liguori, A. Tani and S. ZecchiOrlandini, 1998. Ischemia-reperfusion-induced apoptosis and p53 expression in the course of rat heterotopic heart transplantation. Microvasc. Res., 56: 277-281. 
Nakagawa, T., T. Yokozawa, A. Satoh and H.Y. Kim, 2005. Attenuation of renal ischemia-reperfusion injury by proanthocyanidin-rich extract from grape seeds. J. Nutr. Sci. Vitaminol., 51: 283-286.

Puiggros, F., N. Llopiz, A. Ardevol, C. Blade, L. Arola and M.J. Salvado, 2005. Grape seed procyanidins prevent oxidative injury by modulating the expression of antioxidant enzyme systems. J. Agric. Food Chem., 53: 6080-6086.

Ray, S.D., D. Patel, V. Wong and D. Bagchi, 2000. In vivo protection of DNA damage associated apoptotic and necrotic cell deaths during acetaminophen-induced nephrotoxicity, amiodarone-induced lung toxicity and doxorubicin-induced cardiotoxicity by a novel IH636 grape seed proanthocyanidin extract. Res. Commun. Mol. Pathol. Pharmacol., 107: 137-166.
Sato, M., G. Maulik, P.S. Ray, D. Bagchi and D.K. Das, 1999. Cardioprotective effects of grape seed proanthocyanidin against ischemic reperfusion injury. J. Mol. Cell Cardiol., 31: 1289-1297.

Shi, J., J. Yu, J.E. Pohorly and Y. Kakuda, 2003. Polyphenolics in grape seeds-biochemistry and functionality. J. Med. Food, 6: 291-299.

Wei, R., R. Ding, Y. Wang and L. Tang, 2012. Grape seed proanthocyanidin extract reduces renal ischemia/ reperfusion injuries in rats. Am. J. Med. Sci., 343: 452-457.

Yanarates, O., A. Guven, A. Sizlan, B. Uysal and O. Akgul et al., 2008. Ameliorative effects of proanthocyanidin on renal ischemia/reperfusion injury. Ren. Fail., 30: 931-938. 